\title{
ASYMPTOTIC BEHAVIOR OF EIGENFUNCTIONS OF THE THREE-PARTICLE SCHRÖDINGER OPERATOR. II. CHARGED ONE-DIMENSIONAL PARTICLES
}

\author{
V. S. BUSLAEV AND S. B. LEVIN
}

Dedicated to Ludwig Dmitrievich Faddeev on the occasion of his 75 th birthday

\begin{abstract}
A system of three one-dimensional quantum particles with Coulomb pairwise interaction is treated. A scattered plane wave type asymptotic description at infinity in the configuration space of generalized eigenfunctions is obtained. Though remaining at a heuristic level, the constructions of the paper may serve as a basis for rigorous proofs of the results.
\end{abstract}

\section{§1. INTRODUCTION}

1.1. Scattered plane wave. We consider the Schrödinger differential equation for a system of three identical one-dimensional quantum particles.

We shall need some general geometric definitions. The configuration space of the system (see [1] for the details) is the Euclidean plane $\Gamma$ with the scalar product $\langle.,$. and with 3 distinguished unit vectors $\mathbf{l}_{j}, j=1,2,3$, such that $\mathbf{l}_{2}$ results from $\mathbf{l}_{1}$, and $\mathbf{l}_{3}$ results from $\mathbf{l}_{2}$ by rotation through $2 \pi / 3$ in one and the same direction. Along with these vectors, we consider 3 unit vectors $\mathbf{k}_{j}$ that result from the $\mathbf{l}_{j}$ after rotation through $\pi / 2$ in the direction mentioned above.

A vector on $\Gamma$ will be denoted by $\mathbf{x}$, and its coordinates in the basis $\left(\mathbf{k}_{j}, \mathbf{l}_{j}\right)$ by $\left(x_{j}, y_{j}\right)$. These are classical Jacobi coordinates. Also, vectors denoted by $\mathbf{q}$ with coordinates $\left(k_{j}, p_{j}\right)$ will occur. The difference in the notation indicates that we are dealing with the dual space, identified with $\Gamma$. Let $l_{j}$ denote the subspace spanned by $\mathbf{l}_{j}$. Finally, the symbols $l_{j}^{ \pm}$denote the rays lying on $l_{j}$ and directed along $\pm \mathbf{l}_{j}$. The formula $\langle\mathbf{x}, \mathbf{q}\rangle=$ $\frac{2}{3}\left(x_{1} q_{1}+x_{2} q_{2}+x_{3} q_{3}\right)$ will happen to be useful sometimes.

The group $S$ generated by the reflections $\tau_{j}$ in the lines $l_{j}$ acts on the plane in a natural way. It comprises 6 elements $I, \tau_{1}, \tau_{2}, \tau_{3}, \tau_{2} \tau_{3}, \tau_{2} \tau_{1}$, generically denoted by $\sigma$.

The Schrödinger equation has the form

$$
H \psi=E \psi, \quad H=-\triangle+V(\mathbf{x}), \quad \psi=\psi(\mathbf{x}) \in \mathbb{C} .
$$

Here $\triangle$ is the Laplacian on $\Gamma$ and $E$ is a positive parameter. The potential $V$ has the form

$$
V(\mathbf{x})=v\left(x_{1}\right)+v\left(x_{2}\right)+v\left(x_{3}\right),
$$

where $v(x)$ is a smooth real-valued function (the potential of the two-particle interaction).

2010 Mathematics Subject Classification. Primary 81U10.

Key words and phrases. Quantum scattering, three-particle scattering, Coulomb interaction, onedimensional particles.

Supported by RFBR (grant no. 08-01-00209). 


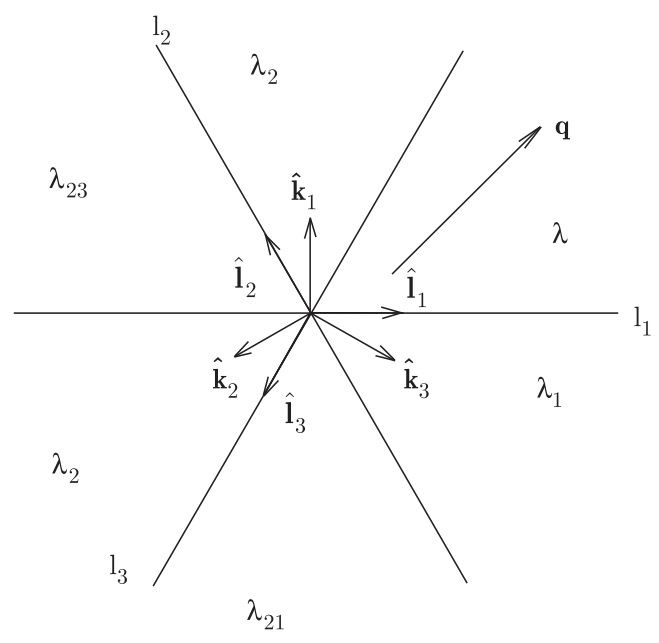

Figure 1

In [1, the case of compactly supported (or rapidly decaying) pairwise potentials was considered, and the function $\psi(\mathbf{x}, \mathbf{q})$ was studied with the following asymptotic behavior as $|\mathbf{x}| \rightarrow \infty$ :

$$
\psi(\mathbf{x}, \mathbf{q})=n(\omega, \mathbf{q}) R^{*}(r, E)+f(\omega, \mathbf{q}) R(r, E)+o\left(r^{-1 / 2}\right) .
$$

Here $r=|\mathbf{x}|, \omega=\frac{\mathbf{x}}{r}, \theta=\frac{\mathbf{q}}{\sqrt{E}}$. We describe the components of the asymptotic formula

$$
n=\sqrt{\frac{2 \pi}{i \sqrt{E}}} \delta(\omega, \theta),
$$

where $\delta(\omega, \theta)$ is the $\delta$-function on the unit circle normalized by the angular measure,

$$
R(r, E)=\frac{1}{r^{1 / 2}} \exp [i \sqrt{E} r]
$$

and ${ }^{*}$ is the symbol of complex conjugation.

The asymptotic formula is understood in the weak sense relative to $\omega$; both $n$ and $f$ are singular distributions.

The first summand in the asymptotic formula coincides with the first (convergent) term in the asymptotics for the plane wave $e^{i\langle\mathbf{q}, \mathbf{x}\rangle}$; therefore, we use the term a scattered plane wave for the solution $\psi(\mathbf{x}, \mathbf{q})$. The convergent term normalizes the solution.

1.2. Coulomb potentials. In the case of pairwise Coulomb potentials, the function $v$ remains smooth but does not decay rapidly any longer. More specifically, we assume that, asymptotically as $|x| \rightarrow \infty$, we have

$$
v(x) \sim \frac{\alpha}{|x|}, \quad \alpha=\text { const }>0 .
$$

As is well known, such a slow decay of $v$ influences the type of a solution crucially. This happens because such potentials influence the asymptotic behavior of a solution at arbitrarily large distances from the centers $x_{j}=0$ of pairwise interactions.

First, the definition of a scattered plane wave changes. However, it suffices to change only the definition of $R$ in the asymptotic description. Namely, $R$ should be replaced 
with the function

$$
\begin{aligned}
R_{c} & =\frac{1}{r^{1 / 2}} \exp [i \sqrt{E} r+i \gamma \ln r], \\
\gamma & =-\frac{\alpha}{2|\mathbf{q}|}\left(\frac{1}{\left|\omega_{1}\right|}+\frac{1}{\left|\omega_{2}\right|}+\frac{1}{\left|\omega_{3}\right|}\right) .
\end{aligned}
$$

After this change, the definition of a scattered plane wave is suitable also in the case of Coulomb potentials.

Yet in this situation no global analog of the plane wave itself exists. But it is possible to consider such an analog inside every sector $\lambda_{\sigma}$ between two neighboring rays $l_{j}^{ \pm}$, where $\sigma$ denotes the element of $S$ that takes the sector $\lambda$ between $l_{1}^{+}$and $l_{3}^{-}$to the sector in question. We denote this analog by $\psi_{\sigma}(\mathbf{x}, \mathbf{q})$; it is given by the formula

$$
\begin{aligned}
\psi_{\sigma}(\mathbf{x}, \mathbf{q}) & =\exp \left[i\langle\mathbf{q}, \mathbf{x}\rangle+i \Delta_{\sigma}(\mathbf{x}, \mathbf{q})\right], \\
\Delta_{\sigma}(\mathbf{x}, \mathbf{q}) & =-\alpha\left(\frac{\operatorname{sgn}\left(x_{1}\right)}{2 k_{1}} \ln \left|x_{1}\right|+\frac{\operatorname{sgn}\left(x_{2}\right)}{2 k_{2}} \ln \left|x_{2}\right|+\frac{\operatorname{sgn}\left(x_{3}\right)}{2 k_{3}} \ln \left|x_{3}\right|\right) .
\end{aligned}
$$

It is easily seen that $\Delta_{\sigma}$ satisfies the equation

$$
2\left\langle\mathbf{q}, \nabla \Delta_{\sigma}\right\rangle+\alpha\left(\frac{1}{\left|x_{1}\right|}+\frac{1}{\left|x_{2}\right|}+\frac{1}{\left|x_{3}\right|}\right)=0
$$

The meaning of the expression suggested here is that it has a discrepancy that decays as a power function as $r \rightarrow \infty$, but decays faster than the Coulomb potential $r^{-1}$. For this, it should be assumed that $\mathbf{q}$ lies outside some angular neighborhood of the subspaces $l_{j}$ and $\mathbf{x}$ tends to infinity remaining outside the same neighborhoods. This property of $\psi_{c}$ is verified by direct substitution in the Schrödinger equation; later we shall discuss this in more detail.

A principal problem is in the proof of the existence and uniqueness of solutions of scattered Coulomb waves type. Also, some procedures should be suggested enabling us to find such a solution numerically.

Here we discuss a partial problem that is expected to lead to this. Namely, we describe the singular part $\psi_{\text {as }}$ of the asymptotic expansion for $\psi$. Technically, this means that we construct a function $\psi_{\text {as }}$ that determines $\psi$ up to an expanding Coulomb circular wave:

$$
\psi-\psi_{\text {as }} \sim g(\omega, \mathbf{q}) R_{c}(r, E),
$$

where $g$ is a smooth (or continuous) function of $\omega$.

The function $\psi_{\text {as }}$ is constructed explicitly. For definiteness, we assume that $\mathbf{q} \in \lambda$ (this is not a restriction) and act in accordance with two criteria:

1 ) the function $\psi_{\text {as }}$ is constructed in such a way that its discrepancy

$$
Q\left[\psi_{\text {as }}\right]=(H-E) \psi_{\text {as }}
$$

decays faster than the Coulomb potential in any direction;

2) the difference

$$
\psi_{\mathrm{as}}(\mathbf{x}, \mathbf{q})-\psi_{\tau_{2}}(\mathbf{x}, \mathbf{q}) \zeta(\omega)
$$

where $\zeta(\omega)$ is a smooth cutoff function supported in a neighborhood of the "opposite" wave vector $-\theta$, behaves asymptotically as the "expanding Coulomb wave"

$$
\sim R_{c} h(\omega, \mathbf{q}) .
$$

So, the problem will be to construct a function $\psi_{\text {as }}$ satisfying 1) and 2). In [1, it was explained how to use such a result for the solution of the principal problem. In [2], it was shown how the result can be used to find a solution $\psi$ numerically if the pairwise potentials decay rapidly. A similar numerical pattern applies to Coulomb potentials without substantial changes. 
In this paper, we construct a near-solution $\psi_{\text {as }}$ that satisfies (2) and has a discrepancy with the following order of decay at infinity:

$$
Q\left[\psi_{\mathrm{as}}\right]=O\left(r^{-\delta}\right), \quad \delta<4 / 3
$$

It is essential that the construction can be refined to yield a near-solution with discrepancy that decays faster than any power of $|\mathbf{x}|^{-1}$.

Also, in 1], the status of the problem in question within related themes was discussed, specifically, within scattering theory for 3 quantum particles, and particularly in the case of a Coulomb interaction. To summarize, the last problem remains the only one in the multiparticle scattering theory for which there is no "stationary approach," necessary for finding parameters of real physical reactions.

Finally, we remind the reader that the case of fast decaying pairwise potentials was studied systematically by L. D. Faddeev as early as in 1963 (see [3]).

Next, note that here we restrict ourselves to nonnegative pairwise potentials $v$. This makes it possible to disregard the effects related to junior spectral channels. Taking them into account is a simpler task than description of processes in senior channels. For simplicity, we also assume that $v(x)$ is an even function and that the particle masses are equal.

We confine ourselves to these short comments, supplemented by the reference to 1 .

The layout of the paper is as follows.

In $\S 2$, we construct a solution $\chi_{j}(\mathbf{x}, \mathbf{q})$ that possesses the required properties in some angular neighborhood of the ray $l_{j}^{ \pm}$.

In $\S 3$, we unite the near-solutions $\chi_{j}$ into a certain ray near-solution $\psi_{R}$. This solution is smooth everywhere except for the rays generated by the vectors $\mathbf{q}_{1}=\tau_{2} \tau_{3} \mathbf{q}$ and $\mathbf{q}_{3}=\tau_{2} \tau_{1} \mathbf{q}$. On these rays, the solution has discontinuities of the first kind.

In $\S 4$, we correct these discontinuities and arrive at a smooth near-solution $\psi_{\text {as }}$ satisfying the criteria 1) and 2).

\section{§2. Asymptotic structure of the field NeAR the Lines $l_{j}$}

2.1. Subdomains. Consider a finite angular neighborhood $V$ of the ray $l_{1}^{+}$; let $V$ be bounded by rays $l_{+}$and $l_{-}$, the first lying inside the sector $\lambda$ and the second inside $\lambda_{\tau_{1}}$. We assume that these rays are symmetric with respect to $l_{1}^{+}$.

Clearly,

$$
x_{2}=-\frac{\sqrt{3}}{2} y_{1}-\frac{1}{2} x_{1}<0, \quad x_{3}=\frac{\sqrt{3}}{2} y-\frac{1}{2} x>0
$$

on $V$. As to the vector $\mathbf{q}$, we agree that $k_{1}>0$.

In this section, $l_{1}$ plays a distinguished role, so we shorten the notation and write $x$ for $x_{1}$ and $y$ for $y_{1}$.

Next, we introduce the immediate neighborhood $V_{0}$ of $l_{1}^{+}$. It contains this ray and lies within the curve $|x|=y^{\nu}, 0<\nu<1$. In this neighborhood, we shall construct a near-solution $\chi(\mathbf{x}, \mathbf{q})$ of the Schrödinger equation whose discrepancy will decay faster than the Coulomb potential; the specific order of decay will be

$$
Q\left[\chi_{0}\right]=O\left(\frac{1+|x|}{y^{2}}\right) .
$$

Also, we consider two domains $V_{ \pm}$lying in $V$. They are symmetric with respect to the ray $l_{1}^{+}$, and $V_{+}$lies in the domain $x>0$ within $l_{+}$and part of the curve $x=y^{\mu}$, $1 / 2<\mu<\nu$, in the upper half-plane. 


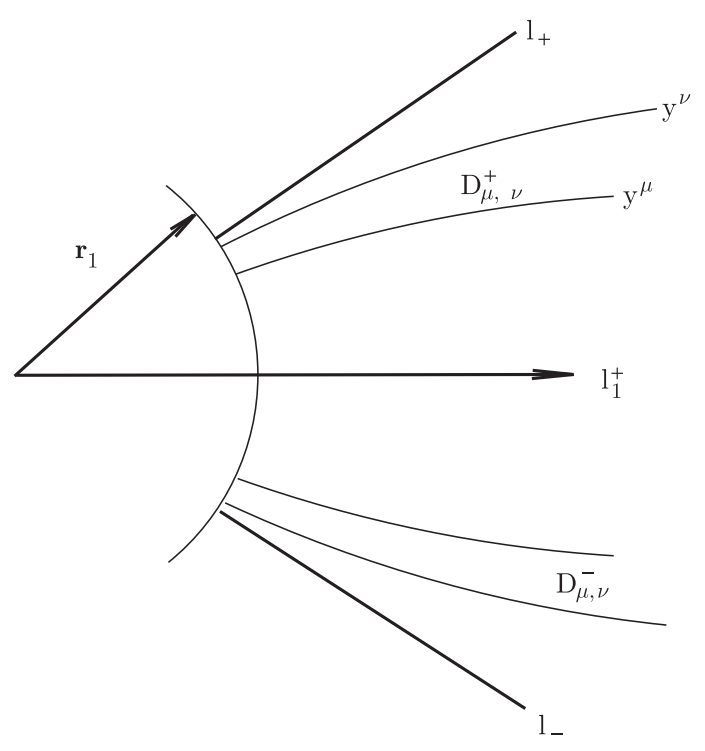

FIGURE 2

We consider the Coulomb waves $\psi_{I}, \psi_{\tau_{1}}$ on these domains. Their discrepancies on these sets have the following order:

$$
Q\left[\psi_{I, \tau_{1}}\right]=O\left(\frac{1}{x^{2}}\right)
$$

that is, they also decay faster than the Coulomb potential.

We combine them in the solutions

$$
\begin{aligned}
& \psi_{+}(\mathbf{x}, \mathbf{q})=s\left(k_{1}\right) \psi_{I}(\mathbf{x}, \mathbf{q}), \\
& \psi_{-}(\mathbf{x}, \mathbf{q})=\psi_{\tau_{1}}(\mathbf{x}, \mathbf{q})+r\left(k_{1}\right) \psi_{\tau_{1}}\left(\mathbf{x}, \tau_{1} \mathbf{q}\right),
\end{aligned}
$$

where $s(k)$ and $r(k)$ are the transition and reflection coefficients for the one-dimensional equation

(see Subsection 2.3).

$$
-\chi^{\prime \prime}+v(x) \chi=k^{2} \chi
$$

Also, we need the sets $D_{\mu, \nu}^{ \pm}, \mu<\nu$ (again, they are symmetric with respect to $l_{1}^{+}$):

$$
D_{\mu, \nu}^{+}=V_{+} \cap V_{0}
$$

In all these domains, we include only points with $r>r_{1}>0$, where $r_{1}$ is sufficiently large.

On these sets, the corresponding near-solutions $\chi_{1}, \psi_{ \pm}$have satisfactory discrepancies; therefore, finally it will be possible to unite them in a near-solution $\chi$ with a satisfactory discrepancy on $V$ :

$$
\chi=\zeta_{+} \psi_{+}+\zeta_{-} \psi_{-}+\zeta_{0} \chi_{1} .
$$

Here $\zeta_{+}, \zeta_{-}$, and $\zeta_{0}$ are the components of a partition of unity

$$
1=\zeta_{+}+\zeta_{-}+\zeta_{0}
$$

subordinate to the covering

$$
V=V_{0} \cup V_{1}^{-} \cup V_{1}^{+} .
$$

We shall assume that these functions depend on the ratio $\rho=\frac{\ln |x|}{\ln y}$ in the regions of nonconstancy. 
The near-solution $\chi$ is an analog of the (exact) solution $\psi$ considered in [1]. It is easy to trace that, in [1], $\psi$ was the principal object, which was used to construct an expression of the type $\psi_{\text {as }}$. In what follows, we use [1] as a pattern.

The remaining part of this section is devoted to the implementation of the above plan.

2.2. Coulomb plane waves near the ray $l_{1}^{+}$. We estimate the discrepancies of Coulomb plane waves:

$$
\begin{aligned}
Q\left[\psi_{\sigma}\right]= & e^{i\langle\mathbf{q}, \mathbf{x}\rangle}\left\{-\Delta e^{i \Delta_{\sigma}}+\left[2\left\langle q, \nabla \Delta_{\sigma}\right\rangle+\alpha\left(\frac{1}{\left|x_{1}\right|}+\frac{1}{\left|x_{2}\right|}+\frac{1}{\left|x_{3}\right|}\right)\right] e^{i \Delta_{\sigma}}\right. \\
& \left.+\left[V-\alpha\left(\frac{1}{\left|x_{1}\right|}+\frac{1}{\left|x_{2}\right|}+\frac{1}{\left|x_{3}\right|}\right)\right] e^{i \Delta_{\sigma}}\right\} \\
= & e^{i\langle\mathbf{q}, \mathbf{x}\rangle}\left\{-\Delta e^{i \Delta_{\sigma}}+\left[V-\alpha\left(\frac{1}{\left|x_{1}\right|}+\frac{1}{\left|x_{2}\right|}+\frac{1}{\left|x_{3}\right|}\right)\right] e^{i \Delta_{\sigma}}\right\} .
\end{aligned}
$$

We have $x_{1}^{2} \ll x_{2}^{2}, x_{3}^{2}$ in the domain in question. Therefore,

$$
\left|Q\left[\psi_{c}^{ \pm}\right]\right| \leq K\left(\frac{1}{x_{1}^{2}}+\frac{1}{x_{2}^{2}}+\frac{1}{x_{3}^{2}}\right) \leq K_{1} \frac{1}{x_{1}^{2}}
$$

This means that, in $V_{ \pm}$, the discrepancies decay at infinity as $y^{-2 \mu}$, that is, faster than the Coulomb potential if $\mu>1 / 2$.

When comparing (in what follows) the near-solutions $\chi_{0}$ and $\psi_{ \pm}$on the domains $D_{\mu, \nu}^{ \pm}$, we should simplify them somewhat in order to ensure that they be mutually close as $y \rightarrow \infty$.

Consider $\psi_{\sigma}$ :

$$
\begin{gathered}
\psi_{\sigma}=e^{i\langle\mathbf{q}, \mathbf{x}\rangle} e^{i \Delta_{\sigma}}, \\
\Delta_{\sigma}=-\alpha\left(\frac{\operatorname{sgn}(x)}{2 k_{1}} \ln |x|+\frac{1}{2 m_{1}} \ln \frac{\sqrt{3}}{2} y-\frac{1}{2 \sqrt{3}}\left(\frac{1}{k_{2}}+\frac{1}{k_{3}}\right) \frac{x}{y}+O\left(\frac{x^{2}}{y^{2}}\right)\right), \\
\frac{1}{m_{1}}=\frac{1}{k_{3}}-\frac{1}{k_{2}} .
\end{gathered}
$$

Let

$$
\psi_{\sigma}^{(s)}=e^{i\langle\mathbf{q}, \mathbf{x}\rangle} e^{-i \alpha\left(\frac{\operatorname{sgn}(x)}{2 k_{1}} \ln |x|+\frac{1}{2 m_{1}} \ln \frac{\sqrt{3}}{2} y\right)}\left[1+i A \frac{x}{y}\right], \quad A=\frac{\alpha}{2 \sqrt{3}}\left(\frac{1}{k_{2}}+\frac{1}{k_{3}}\right) .
$$

Thus,

$$
\psi_{\sigma}=\psi_{\sigma}^{(s)}\left(1+O\left(\frac{x^{2}}{y^{2}}\right)\right)
$$

It is easily seen that

$$
Q\left[\psi_{\sigma}^{(s)}\right]=O\left(\frac{1}{x^{2}}+\frac{x}{y^{2}}\right)
$$

Now, using the same formulas that describe $\psi_{ \pm}$in terms of $\psi_{\sigma}$, we can construct $\psi_{ \pm}^{(s)}$ in terms of $\psi_{\sigma}^{(s)}$.

In the sequel we shall need some information about the solutions of the scattering problem for the one-dimensional Schrödinger equation. 
2.3. Solution of the scattering problem on the axis. Consider a solution $\xi(x, k)$ of the scattering problem for the one-dimensional Schrödinger equation

$$
-\xi^{\prime \prime}+v(x) \xi=k^{2} \xi, \quad x, k \in \mathbb{R} .
$$

To describe it in more specific terms, we put

$$
\xi_{c}(x, k)=\exp \left[i k x-\frac{i \alpha}{2 k} \operatorname{sgn}(x) \ln |x|\right] .
$$

Using this notation, we can say that $\xi(x, k)$ is characterized by the following asymptotic behavior as $|x| \rightarrow \infty$ :

$$
\begin{aligned}
\xi(x, k) & \sim s(k) \xi_{c}(x, k), \quad k x \rightarrow+\infty, \\
\chi(x, k) & \sim \xi_{c}(x, k)+r(k) \xi_{c}^{*}(x, k), \quad k x \rightarrow-\infty .
\end{aligned}
$$

Since the potential is even, we have

$$
\xi(-x,-k)=\xi(x, k)
$$

moreover, $s(k)=s(|k|)$ and $r(k)=r(|k|)$.

2.4. Separation of variables in the immediate neighborhood of the ray $l_{1}^{+}$. In the immediate neighborhood of the ray, where $|x| \ll y, y \gg 1$, the potential $V$ can be simplified as follows:

$$
V(\mathbf{x}) \sim v(x)+2 v\left(\frac{\sqrt{3}}{2} y\right) .
$$

Retaining only the senior order as $y \rightarrow+\infty$ in the potential, we arrive at the equation

$$
-\triangle \chi_{0}+\left[v(x)+\frac{4 \alpha}{\sqrt{3}|y|}\right] \chi_{0}=E \chi_{0} .
$$

It admits separation of variables and, consequently, has a solution of the form

$$
\chi_{0}(\mathbf{x}, \mathbf{q})=\xi(x, k) f(y, p),
$$

where $\xi(x, k)$ and $f(y, p)$ are solutions of the ordinary differential equations

$$
-\xi^{\prime \prime}+v \xi=k^{2} \xi, \quad-f^{\prime \prime}+\frac{4 \alpha}{\sqrt{3}|y|} f=p^{2} f .
$$

Here $k^{2}+p^{2}=E$.

For $\xi$, we agree to take the solution of the one-dimensional scattering problem. As to $f$, we replace it with the senior term in the asymptotics as $y \rightarrow+\infty$. Moreover, as in [1, we retain only one summand in the expression for $f$ :

$$
f(y, p) \sim \exp \left[i p y-i \frac{2 \alpha}{\sqrt{3} p} \ln y\right] .
$$

Thus, at this stage we suggest the following approximation for $\chi_{0}(\mathbf{x}, \mathbf{q})$ :

$$
\chi_{0}(\mathbf{x}, \mathbf{q})=\xi(x, k) \exp \left[i p y-i \frac{2 \alpha}{\sqrt{3} p} \ln y\right] .
$$

However, this does not finish the construction of a near-solution in an immediate neighborhood of the ray. We have constructed only a zero approximation, whose discrepancy does not decay sufficiently fast at infinity (it only decays as the Coulomb potential). A satisfactory approximation looks like the following:

$$
\chi_{1}=\left(\xi(x, k)+\frac{B}{y} \xi_{k}(x, k)\right) \exp \left[i p y+i \gamma_{0} \ln y\right],
$$

where the numbers $B_{0}$ and $\gamma$ are to be determined. 
We substitute the suggested Ansatz in the equation:

$$
\begin{aligned}
Q\left[\chi_{1}\right]=\exp \left[i p y+i \gamma_{0} \ln y\right]\{ & {\left[\left(i p+i \gamma_{0} \frac{1}{y}\right)^{2}-i \gamma_{0} \frac{1}{y^{2}}\right]\left(\xi+\frac{B}{y} \xi_{k}\right) } \\
& -2 \frac{B}{y^{2}} \xi_{k}\left(i p+i \gamma_{0} \frac{1}{y}\right)+\left[\xi_{x x}+\frac{B}{y} \xi_{k x x}+\frac{2 B}{y^{3}} \xi_{k}\right] \\
+ & \left.\left(p^{2}+k^{2}-v(x)-\frac{\beta}{y}+O\left(\frac{x^{2}}{y^{3}}\right)\right)\left(\xi+\frac{B}{y} \xi_{k}\right)\right\}, \quad \beta=\frac{4 \alpha}{\sqrt{3}} .
\end{aligned}
$$

Let $y \rightarrow+\infty$. The differential equation for $\xi$ implies mutual cancellation of the terms in the discrepancy that do not tend to 0 separately. The terms that decay as $1 / y$ also cancel mutually because

$$
-\xi_{k x x}+v(x) \xi_{k}-k^{2} \xi_{k}=2 k \xi
$$

provided we put

$$
\beta=-2 p \gamma_{0}-2 k B .
$$

The last relation can be viewed as an equation for the parameters $B$ and $\gamma_{0}$.

As a result, the discrepancy is estimated as follows:

$$
Q\left[\chi_{1}\right]=O\left(\frac{1+|x|}{y^{2}}\right) .
$$

The growth of the estimate in $x$ has arisen because the derivative $\xi_{k}(x, k)$ grows linearly.

2.5. Asymptotic behavior of the field $\chi_{1}$ for $|x| \gg 1$. So, suppose $k_{1}>0$ and $\mathbf{x} \in D_{\mu, \nu}^{ \pm}$(thus, $|x| \gg 1$ ).

For $x>0$, at the senior order the solution $\chi_{1}$ takes the form

$$
\chi_{1} \sim s\left(k_{1}\right) e^{\langle\mathbf{q}, \mathbf{x}\rangle} e^{\left[-\frac{i \alpha}{2 k_{1}} \ln |x|\right]}\left(1-i B \frac{x}{y}\right)+O\left(\frac{1}{x}\right) .
$$

Comparing this with the formula for $\psi_{+}^{(s)}$, we see that, obviously, it should be taken to be

$$
B=A=\frac{\alpha}{2 \sqrt{3}}\left(\frac{1}{k_{2}}+\frac{1}{k_{3}}\right) .
$$

Now, equation (11) allows us to calculate $\gamma_{0}$ :

$$
\gamma_{0}=\alpha \frac{1}{2 m_{1}} .
$$

We have used the formula

$$
p=\frac{1}{\sqrt{3}}\left(k_{2}-k_{3}\right) .
$$

Adopting these expressions for the constants, on $D_{\mu, \nu}^{(+)}$we obtain

$$
\chi_{1}=\psi_{+}^{(s)}+O\left(\frac{1}{x}\right) .
$$

Similar arguments are applicable for $x<0$, yielding

$$
\chi_{1}=\psi_{-}^{(s)}+O\left(\frac{1}{x}\right)
$$

on $D_{\mu, \nu}^{(-)}$. 
2.6. The outcome. We have agreed to define a near-solution $\chi$ on $V$ by the formula

$$
\chi=\zeta_{+} \psi_{+}+\zeta_{-} \psi_{-}+\zeta_{0} \chi_{1} .
$$

Now, we are in a position to finalize the construction of a near-solution $\chi$ in the entire angular neighborhood $V$ of $l_{1}^{+}$in order to satisfy the criteria 1) and 2) stated before. Criterion 2) is met indeed for fairly obvious reasons, so we only discuss the discrepancy decay, i.e., criterion 1 ).

For $x>0$ we have

$$
\begin{aligned}
Q[\chi] & =Q_{1}[\chi]+Q_{2}[\chi], \quad Q_{1}[\chi]=Q\left[\chi_{1}\right] \zeta_{0}+Q\left[\psi_{+}\right] \zeta_{+}, \\
Q_{2}[\chi] & =-\left(\Delta \zeta_{0}\right)\left(\chi_{1}-\psi_{+}\right)-2\left(\nabla \zeta_{0}, \nabla\left(\chi_{1}-\psi_{+}\right)\right) .
\end{aligned}
$$

For $x<0$ we have

$$
\begin{aligned}
Q[\chi] & =Q_{1}[\chi]+Q_{2}[\chi], \quad Q_{1}[\chi]=Q\left[\chi_{1}\right] \zeta_{0}+Q\left[\psi_{-}\right] \zeta_{-}, \\
Q_{2}[\chi] & =-\left(\Delta \zeta_{0}\right)\left(\chi_{1}-\psi_{-}\right)-2\left(\nabla \zeta_{0}, \nabla\left(\chi_{1}-\psi_{-}\right)\right) .
\end{aligned}
$$

Consider the case of $x>0$. The required estimate for $Q_{1}[\chi]$ follows immediately from the results of Subsections 2.2 and 2.4. It should be taken into account that the first summand does not vanish only on $V_{0}$, where

$$
Q_{1}[\chi]=O\left(\frac{1+x}{y^{2}}\right), \quad \mathbf{x} \in V_{0},
$$

and similarly, the second summand does not vanish only on $V_{+}$, where

$$
Q_{1}[\chi]=O\left(\frac{1}{x^{2}}\right), \quad \mathbf{x} \in V_{+} .
$$

We turn to $Q_{2}[\chi]$. This summand is nonzero only on $D_{\mu, \nu}^{+}$. By the choice of cutoff functions, it is immediate that $\nabla \zeta_{0}=O\left(x^{-1}\right), \Delta \zeta_{0}=O\left(|x|^{-2}\right)$. Next, $\nabla\left(\chi_{1}-\psi_{+}\right)=$ $O\left(x^{-1}\right)$ by the formulas in Subsection 2.5. Therefore,

$$
Q_{2}[\chi]=O\left(\frac{1}{x^{2}}\right), \quad \mathbf{x} \in D_{\mu, \nu}^{+} .
$$

Consequently, for $x>0$, the discrepancy is estimated as follows:

$$
\begin{aligned}
& Q[\chi]=O\left(\frac{1}{x^{2}}\right), \quad \mathbf{x} \in V_{+}, \\
& Q[\chi]=O\left(\frac{1+x}{y^{2}}\right), \quad \mathbf{x} \in V_{0}, \\
& Q[\chi]=O\left(\frac{1+x}{y^{2}}+\frac{1}{x^{2}}\right), \quad \mathbf{x} \in V_{0} \cup V_{+} .
\end{aligned}
$$

The same discrepancy estimates are true for $x<0$.

Now it is easily seen that, on $V$, the discrepancy admits the estimate

$$
Q[\chi]=O\left(\frac{y^{\nu}}{y^{2}}+\frac{1}{y^{2 \mu}}\right) .
$$

If we take $\mu$ and $\nu$ close to $2 / 3$ with $\mu<\nu$, the discrepancy will be of the following order:

$$
Q[\chi]=O\left(y^{-\delta}\right), \quad \delta<4 / 3 .
$$

Thus, definitely, the discrepancy decays faster than the Coulomb potential, and we have achieved our goal. 


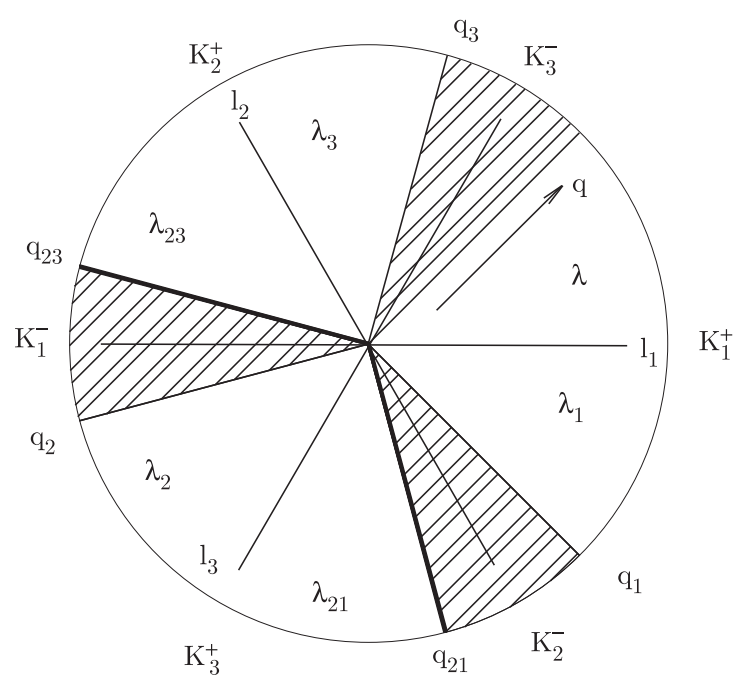

FiguRE 3

\section{$\S 3$. FORMUlas FOR $\psi_{R}$}

3.1. Construction of $\psi_{R}$. In this and in the next section we complete the construction of the functions $\psi_{\text {as }}$. The present section is devoted to a description of a ray approximation $\psi_{R}$ to that function; $\psi_{R}$ will not meet our requirements only on two among the six rays $q_{\sigma}$ directed along the vectors $\mathbf{q}_{\sigma}, \sigma \in S$. In the next section, we correct the field on these two rays, which will finish the construction of the field $\psi_{\text {as }}$. Recall that there is precisely one ray $\mathbf{q}_{\sigma}$ in each sector $\lambda_{\sigma}, \sigma \in S$.

To a large extent, the content of the two sections is a repetition of [1]. Moreover, in the present section not only do we follow the ideas of [1, but in fact we lean upon the proofs therein.

On $V$, outside a disk of sufficiently large radius, we have already constructed a nearsolution $\chi$ with discrepancy decaying faster than the Coulomb potential at infinity. Starting with this point, we omit the lower index in the notation for this solution. We extend this solution to the set $\widehat{V}$ obtained by supplementing $V$ with the domain symmetric to $V$ relative to the line generated by $\mathbf{k}_{1}$. This is done with the help of the formula

$$
\chi\left(x, y, k_{1}, l_{1}\right)=\chi\left(x,-y, k_{1},-l_{1}\right) .
$$

We retain the notation $\chi$ for the field on $\widehat{V}$.

As in [1, we introduce the functions

$$
\chi_{j}(\mathbf{x}, \mathbf{q})=\chi\left(x_{j}, y_{j}, k_{j}, l_{j}\right) .
$$

The rays $q_{\sigma}$ give rise to 6 new sectors $K_{j}^{ \pm}$between them.

Each of these sectors contains a unique ray $l_{j}^{ \pm}$, the indices of which are also used to mark the sector $K$ in question. We describe a ray approximation $\psi_{R}$ to the field $\psi_{\text {as }}$ in the sectors $K_{j}^{ \pm}$.

$$
\text { Sector } K_{1}^{+}: \psi_{R}=\chi_{1}^{+} \text {, }
$$

$$
\chi_{1}^{+}(\mathbf{x}, \mathbf{q})=\chi_{1}(\mathbf{x}, \mathbf{q}) s_{2} s_{3} .
$$

We have used the notation $s_{j}=s\left(k_{j}\right), r_{j}=r\left(k_{j}\right)$.

$\underline{\text { Sector } K_{3}^{-}:} \psi_{R}=\chi_{3}^{-}$,

$$
\chi_{3}^{-}(\mathbf{x}, \mathbf{q})=\chi_{3}(\mathbf{x}, \mathbf{q}) s_{2} s_{1}
$$


Sector $K_{2}^{+}: \psi_{R}=\chi_{2}^{+}$,

$$
\chi_{2}^{+}(\mathbf{x}, \mathbf{q})=\chi_{2}(\mathbf{x}, \mathbf{q}) s_{1}+\chi_{2}\left(\mathbf{x}, \tau_{3} \mathbf{q}\right) s_{2} r_{3} .
$$

Sector $K_{2}^{-}: \psi_{R}=\chi_{2}^{-}$,

$$
\chi_{2}^{-}(\mathbf{x}, \mathbf{q})=\chi_{2}(\mathbf{x}, \mathbf{q}) s_{3}+\chi_{2}\left(\mathbf{x}, \tau_{1} \mathbf{q}\right) s_{2} r_{1} .
$$

Sector $K_{1}^{-}: \psi_{R}=\chi_{1}^{-}$,

$$
\chi_{1}^{-}(\mathbf{x}, \mathbf{q})=\chi_{1}(\mathbf{x}, \mathbf{q})+\chi_{1}\left(\mathbf{x}, \tau_{2} \mathbf{q}\right) r_{2} s_{1}+\chi_{1}\left(\mathbf{x}, \tau_{3} \tau_{1} \mathbf{q}\right) r_{2} r_{1}+\chi_{1}\left(\mathbf{x}, \tau_{3} \mathbf{q}\right) r_{3} .
$$

Sector $K_{3}^{+}: \psi_{R}=\chi_{3}^{+}$,

$$
\chi_{3}^{+}(\mathbf{x}, \mathbf{q})=\chi_{3}(\mathbf{x}, \mathbf{q})+\chi_{3}\left(\mathbf{x}, \tau_{2} \mathbf{q}\right) r_{2} s_{3}+\chi_{3}\left(\mathbf{x}, \tau_{1} \tau_{3} \mathbf{q}\right) r_{2} r_{3}+\chi_{3}\left(\mathbf{x}, \tau_{1} \mathbf{q}\right) r_{1} .
$$

The field $\psi_{R}$ is defined by the formula

$$
\psi_{R}=\theta_{1}^{+} \chi_{1}^{+}+\theta_{3}^{-} \chi_{3}^{-}+\theta_{2}^{+} \chi_{2}^{+}+\theta_{2}^{-} \chi_{2}^{-}+\theta_{1}^{-} \chi_{1}^{-}+\theta_{3}^{+} \chi_{3}^{+} .
$$

We have used the notation $\theta_{j}^{( \pm)}$for the characteristic function of the corresponding sector $K_{j}^{ \pm}$,

$$
\theta_{1}^{+}+\theta_{3}^{-}+\theta_{2}^{+}+\theta_{2}^{-}+\theta_{1}^{-}+\theta_{3}^{+}=1 .
$$

The last formula leaves open the question about the magnitude of the fields on the borders of the sectors. In the next section we shall see that on all borders except for the rays $q_{1}$ and $q_{2}$ along the vectors $\mathbf{q}_{1}$ and $\mathbf{q}_{2}$ the field constructed above is smooth.

3.2. Smoothness properties of $\psi_{R}$. Passing to the discussion of the smoothness of the ray approximation $\psi_{R}$ in the vicinity of the border of two sectors of the form $K_{j}^{ \pm}$, first we observe that, on the two sides from this border and near it, $\psi_{R}$ reduces to finite linear combinations of plane waves provided the potentials decay rapidly. The coefficients of these linear combinations are determined by the transition and reflection coefficients of the one-dimensional problem. Thus, on the two sides of the border, the field is completely determined by the corresponding collections of wave vectors and by these coefficients. In [1, it was shown that these objects pertaining to different sides of one border coincide on 4 borders among 6 . On the two remaining borders, $q_{1}$ and $q_{2}$, there are jumps of coefficients of plane waves with wave vector directed along the border.

In the case of Coulomb potentials, the fields near a border are linear combinations of Coulomb plane waves. A Coulomb plane wave differs from the usual one by the factor $\exp \left[i \Delta_{\sigma}\right]$. Now this factor depends not only on the wave vector of the corresponding plane wave, but also on the signs of the variables $x_{j}$ near the border. But surely these signs are the same on the two sides of the border. Thus, again, the collections of Coulomb plane waves coincide. The coefficients of them also coincide because they are determined by the sequence of "transitions" and "reflections" that the wave vector of the incoming wave undergoes in the process of transformation to the wave vector in question. Therefore, on all borders except $q_{1}$ and $q_{2}$, the ray fields are continuous again.

As was shown in [1, the discontinuous part of the ray field near $q_{1}$ is given by

$$
j_{1}=\left(R_{1} \theta_{2}^{+}+R_{2} \theta_{1}^{-}\right) e^{i\left\langle\mathbf{q}_{1}, \mathbf{x}\right\rangle} .
$$

Here $R_{1}=r_{1} s_{2} r_{3}, \quad R_{2}=r_{3} r_{2} s_{1}+s_{3} r_{2} r_{1}$.

Similarly, the discontinuous part of the ray field near $q_{2}$ is given by

$$
j_{3}=\left(R_{1} \theta_{2}^{-}+R_{2} \theta_{3}^{+}\right) e^{i\left\langle\mathbf{q}_{3}, \mathbf{x}\right\rangle} .
$$

In the case of Coulomb potentials, we must replace the plane wave components of these solutions by Coulomb plane waves, to be denoted by $\psi_{1}\left(\mathbf{x}, \mathbf{q}_{1}\right), \psi_{1}=\psi_{\tau_{2} \tau_{3}}$, and $\psi_{3}\left(\mathbf{x}, \mathbf{q}_{3}\right), \psi_{3}=\psi_{\tau_{2} \tau_{1}}$, respectively. 


\section{§4. Smoothing of Discontinuous Solutions}

So, near $q_{1}$ we deal with the discontinuous near-solution

$$
J_{1}=\left(R_{1} \theta_{2}^{+}+R_{2} \theta_{1}^{-}\right) \psi_{1}\left(\mathbf{x}, \mathbf{q}_{1}\right) .
$$

Near $q_{3}$, we deal with the discontinuous near-solution

$$
J_{3}=\left(R_{1} \theta_{2}^{-}+R_{2} \theta_{3}^{+}\right) \psi_{3}\left(\mathbf{x}, \mathbf{q}_{3}\right) .
$$

We discuss a procedure of smoothing $J_{1}$. To begin with, we introduce polar coordinates $r, \eta$ in the sector in question, assuming that $\eta \in[0, \pi / 3], \eta=0 \sim l_{2}^{+}$. Let a function $f$ be supported inside the interval $[0, \pi / 3]$.

To this function, we apply the projections $P_{ \pm}$onto the spaces of functions analytic in the upper or lower half-plane:

$$
\left(P_{ \pm} f\right)(\eta)=\frac{ \pm 1}{2 \pi i} \int_{\mathbb{R}} \frac{d \eta^{\prime}}{\eta^{\prime}-(\eta \pm i 0)} f\left(\eta^{\prime}\right) .
$$

Our goal is to replace discontinuous near-solutions by certain superpositions of Coulomb plane waves, which will be near-solutions with a satisfactory discrepancy. Moreover, we want that, as $\mathbf{x}$ goes away from the discontinuity ray $q_{1}$, these superpositions approach the corresponding Coulomb plane wave components of the ray approximation $\psi_{R}$.

Consider the functions

$$
\phi_{1}^{( \pm)}=\frac{ \pm 1}{2 \pi i} \int_{\mathbb{R}} \frac{d \eta^{\prime}}{\eta^{\prime}-\left(\eta_{0} \pm i 0\right)} f\left(\eta^{\prime}\right) \psi_{1}\left(\mathbf{x}, \eta^{\prime}\right) .
$$

For $f$, we take a function equal to 1 on $[\alpha, \beta], 0<\alpha<\beta<\pi / 3$, and to zero outside $[0, \pi / 3]$. Next, $\psi_{1}\left(\mathbf{x}, \eta^{\prime}\right)=\psi_{1}(\mathbf{x}, \mathbf{q})$, where for $\mathbf{q}$ we employ the polar coordinates $\left(\sqrt{E}, \eta^{\prime}\right)$. These integrals are superpositions of Coulomb plane waves, so they are nearsolutions of the Schrödinger equation with discrepancies satisfying the same estimates as the Coulomb plane waves themselves. Consequently,

$$
Q\left[\phi_{1}^{( \pm)}\right]=O\left(y_{2}^{-2}\right) .
$$

We study the asymptotic behavior of these integrals as $r \rightarrow \infty$ for $\eta \in(\alpha, \beta)$. The method of calculating the asymptotics of the integrals is fairly simple. We write the integrals in a more explicit form:

$$
\phi_{1}^{( \pm)}=\frac{ \pm 1}{2 \pi i} \int_{\mathbb{R}} \frac{f\left(\eta^{\prime}\right) d \eta^{\prime}}{\eta^{\prime}-\left(\eta_{0} \pm i 0\right)} e^{\left[i r \sqrt{E} \cos \left(\eta^{\prime}-\eta\right)+i \Delta\left(\mathbf{x}, \eta^{\prime}\right)\right]},
$$

where $\mathbf{q}$ is the vector whose polar coordinates are $\sqrt{E}, \eta^{\prime}$. Also, we detail the structure of the function $\Delta\left(\mathbf{x}, \eta^{\prime}\right)$ :

$$
\begin{aligned}
\Delta\left(\mathbf{x}, \eta^{\prime}\right) & =\ln r \gamma_{1}\left(\mathbf{q}\left(\eta^{\prime}\right)\right)+\gamma_{2}\left(\omega, \mathbf{q}\left(\eta^{\prime}\right)\right) \\
\gamma_{1} & =-\alpha\left(\frac{1}{2 k_{1}\left(\eta^{\prime}\right)}+\frac{1}{2 k_{2}\left(\eta^{\prime}\right)}-\frac{1}{2 k_{3}\left(\eta^{\prime}\right)}\right) \\
\gamma_{2} & =-\alpha\left(\frac{1}{2 k_{1}\left(\eta^{\prime}\right)} \ln \left|\left(\omega, \mathbf{k}_{1}\right)\right|+\frac{1}{2 k_{2}\left(\eta^{\prime}\right)} \ln \left|\left(\omega, \mathbf{k}_{2}\right)\right|-\frac{1}{2 k_{3}\left(\eta^{\prime}\right)} \ln \left|\left(\omega, \mathbf{k}_{3}\right)\right|\right) .
\end{aligned}
$$

Clearly, the integrand has a stationary point $\eta$ and a pole $\eta_{0}$. The asymptotic behavior of the integral depends on the mutual position of these points. If $r^{1 / 2}\left|\eta-\eta_{0}\right| \rightarrow \infty$, the contributions of the stationary point and the pole can be separated and generate an expanding Coulomb wave (with a certain amplitude) and a plane Coulomb wave. More specifically, for $\eta_{0} \lessgtr \eta$ we have

$$
\phi_{1}^{( \pm)} \sim \psi_{1}\left(\mathbf{x}, \mathbf{q}_{1}\right)+\frac{ \pm e^{-i 3 / 4 \pi}}{\sqrt{2 \pi|\mathbf{q}|}} \frac{1}{\eta-\eta_{0}} e^{i \gamma_{2}(\omega, \mathbf{q}(\eta))} R_{c} .
$$


The opposite combination of the solution's indices leads to the situation in which the poles do not contribute to the asymptotics, and the integrals converge asymptotically to circular Coulomb waves, which play the role of an error in our constructions: for $\eta_{0} \lessgtr \eta$ we have

$$
\phi_{1}^{(\mp)} \sim \frac{\mp e^{-i 3 / 4 \pi}}{\sqrt{2 \pi|\mathbf{q}|}} \frac{1}{\eta-\eta_{0}} e^{i \gamma_{2}(\omega, \mathbf{q}(\eta))} R_{c} .
$$

These formulas show that for $\mathbf{x}$ close to $q_{1}$ it is possible to replace $\theta_{1}^{+} \psi_{1}$ by the nearsolution $\phi_{1}^{+}$, and $\theta_{1}^{-} \psi_{1}$ by the near-solution $\phi_{1}^{-}$. In the next subsection, we describe formulas that realize this idea.

4.1. Smooth near-solutions in the sectors $\lambda_{1}, \lambda_{3}$. On the axis $\eta$, we consider four points $\eta_{1}^{(1)}, \eta_{2}^{(1)}, \eta_{3}^{(1)}$, and $\eta_{4}^{(1)}$ subject to the condition

$$
0<\alpha<\eta_{1}^{(1)}<\eta_{2}^{(1)}<\eta_{0}<\eta_{3}^{(1)}<\eta_{4}^{(1)}<\beta<\pi / 3 .
$$

Consider the covering of the interval $0, \pi / 3$ by the half-intervals $\left(0, \eta_{2}^{(1)}\right),\left(\eta_{1}^{(1)}, \eta_{4}^{(1)}\right)$, $\left(\eta_{3}^{(1)}, \pi / 3\right)$. Next, consider a partition of unity

$$
1=\zeta_{1}^{(1)}+\zeta_{2}^{(1)}+\zeta_{3}^{(1)}
$$

subordinate to this covering. We assume that cutoff functions depend on the polar angle.

Now, we replace the discontinuous part of the field $J_{1}$ in the sector in question by the following near-solution $\widetilde{J}_{1}$ :

$$
\widetilde{J}_{1}=\zeta_{1}^{(1)} J_{1}+\zeta_{3}^{(1)} J_{1}+\zeta_{2}^{(1)}\left(R_{1} \phi_{1}^{(+)}+R_{2} \phi_{1}^{(-)}\right) .
$$

In the sector $\lambda_{3}$, we repeat almost literally the above smoothing constructions for $\lambda_{1}$, obtaining an expression similar to $\widetilde{J}_{1}$ for a smooth near-solution $\widetilde{J}_{3}$ in $\lambda_{3}$ :

$$
\widetilde{J}_{3}=\zeta_{1}^{(3)} J_{3}+\zeta_{3}^{(3)} J_{3}+\zeta_{2}^{(3)}\left(R_{1} \phi_{3}^{(+)}+R_{2} \phi_{3}^{(-)}\right) .
$$

Again, here we assume that the sector is endowed with polar coordinates $r, \eta, \eta \in$ $(0, \pi / 3)$ in such a way that $\eta=0 \sim l_{3}^{(+)}$. As before, we introduce a point $\eta_{0}$ corresponding to the vector $\mathbf{q}_{3}$. Next, we introduce points $\alpha, \beta$ and $\eta_{i}^{(3)}$ much as for the sector $\lambda_{1}$. A covering and a partition of unity come into play after that. Precisely the components of this partition of unity occur in the preceding formula.

It remains to describe $\phi_{3}^{( \pm)}$. The only object involved in the definition of $\phi_{3}^{( \pm)}$that requires an explanation is a Coulomb wave $\psi_{3}\left(\mathbf{x}, \eta^{\prime}\right)$ similar to $\psi_{1}\left(\mathbf{x}, \eta^{\prime}\right)$. In the analytic expression, the difference is in the collection of signs only: $\operatorname{sgn}\left(x_{1}\right)=-1, \operatorname{sgn}\left(x_{2}\right)=1$, $\operatorname{sgn}\left(x_{3}\right)=1$.

It remains to find the rate of decay of the discrepancies for $\widetilde{J}_{1}$ and $\widetilde{J}_{3}$. Consider the discrepancy of $\widetilde{J}_{1}$ :

$$
Q\left[\widetilde{J}_{1}\right]=Q\left[\zeta_{1}^{(1)} J_{1}\right]+Q\left[\zeta_{3}^{(1)} J_{1}\right]+Q\left[\zeta_{2}^{(1)} R_{1} \phi_{1}^{(+)}\right]+Q\left[\zeta_{2}^{(1)} R_{2} \phi_{1}^{(-)}\right] .
$$

In the regions of constancy of the cutoff functions, the question reduces to the discrepancies of $J_{1}$ or $\phi_{1}^{( \pm)}$. Moreover, $J_{1}$ comes into play only on a set where it is smooth. Therefore, on the constancy sets of the cutoff functions, the discrepancies are of the order of $O\left(|\mathbf{x}|^{-2}\right)$.

But on the nonconstancy sets for the cutoff functions, the functions $\phi_{1}^{( \pm)}$reduce to their asymptotics. Consider, for instance, the interval $\left(\eta_{1}^{(1)}, \eta_{2}^{(1)}\right)$. On this interval, the field reduces to the sum of the Coulomb plane wave $\psi_{1}$ and the expanding Coulomb wave

$$
G=g(\omega, \mathbf{q}) R_{c},
$$


with smooth amplitude multiplied by $\zeta_{2}^{(1)}$. The discrepancy of the plane Coulomb wave is $O\left(|\mathbf{x}|^{-2}\right)$. Finally, the discrepancy of the product is given by the formula

$$
Q\left[\zeta_{2}^{(1)} G\right]=-\left(\Delta \zeta_{2}^{(1)}\right) G-2\left\langle\nabla \zeta_{2}^{(1)}, \nabla G\right\rangle+\zeta_{2}^{(1)} Q[G] .
$$

It is easily seen that this discrepancy is of the order of $O\left(|\mathbf{x}|^{-5 / 2}\right)$.

This formula is the final confirmation of the estimate

$$
Q\left[\widetilde{J}_{1}\right]=O\left(|\mathbf{x}|^{-2}\right) .
$$

A similar formula is valid for $Q\left[\widetilde{J}_{3}\right]$. Thus, the construction of $\psi_{\text {as }}$ is complete:

$$
\psi_{\text {as }}=\psi_{R}
$$

everywhere except for the sectors $\lambda_{1}$ and $\lambda_{3}$, and

$$
\psi_{\mathrm{as}}=\psi_{R}+\left(\widetilde{J}_{i}-J_{i}\right)
$$

in these sectors. In any direction, we have the following uniform estimate for the discrepancy:

$$
Q\left[\psi_{\mathrm{as}}\right]=O\left(|\mathbf{x}|^{-\delta}\right), \quad \delta<4 / 3 .
$$

We summarize the results in a theorem.

Theorem 1. The function $\psi_{\mathrm{as}}$ constructed above possesses the following properties:

1) its discrepancy decays at infinity faster than the Coulomb potential and has the order of $O\left(|\mathbf{x}|^{-\delta}\right), \delta<4 / 3$;

2) the difference $\psi_{\mathrm{as}}(\mathbf{x}, \mathbf{q})-\psi_{\tau_{2}}(\mathbf{x}, \mathbf{q}) \zeta(\omega)$, in which $\zeta(\omega)$ is a smooth cutoff function with support in a neighborhood of the "opposite" wave vector $-\mathbf{q}$, behaves asymptotically as an "expanding Coulomb wave" $\sim R_{c} h(\omega, \mathbf{q})$.

Also, we strongly believe that the following claim is true.

Claim. The Schrödinger equation has a solution $\psi$ with the following asymptotic behavior:

$$
\psi-\psi_{\text {as }} \sim g(\omega, \mathbf{q}) R_{c}(r, E)
$$

where $g$ is a continuous function of $\omega$.

We think that the above theorem is an important step towards the proof of the claim.

\section{REFERENCES}

[1] V. S. Buslaev and S. B. Levin, Asymptotic behavior of the eigenfunctions of the many-particle Schrödinger operator. I. One-dimensional particles, Spectral Theory of Differential Operators, Amer. Math. Soc. Transl. (2), vol. 225, Amer. Math. Soc., Providence, RI, 2008, pp. 55-71. MR2509775 (2010k:81292)

[2] V. S. Buslaev, S. B. Levin, P. Neittaannmäki, and T. Ojala, New approach to numerical computation of the eigenfunctions of the continuous spectrum of three-particle Schrödinger operator. I. Onedimensional particles, short-range pair potentials, arXiv:0909.4529v1 [math-ph], (2009).

[3] L. D. Faddeev, Mathematical aspects of the three-body problem in the quantum scattering theory, Trudy Mat. Inst. Steklov. 69 (1963), 122 pp.; English transl., Daniel Davey and Co., Inc., New York, 1965. MR0163695(29:995) MR0221828(36:4880)

Department of Physics, St. Petersburg State University, Ul'yanovskaya 3, St. Petersburg 198504, RUSSIA

E-mail address: vbuslaev@gmail.com

Department of Physics, St. Petersburg State University, Ul’yanovskaya 3, St. Petersburg 198504, RUSSIA

Received 11/DEC/2009

Translated by S. V. KISLYAKOV 